\title{
Request for clarification from Ruiter et al regarding 'Risk of cancer in patients on insulin glargine and other insulin analogues in comparison with those on human insulin: results from a large population-based follow-up study'
}

\author{
B. Carstensen
}

Received: 20 October 2011 / Accepted: 7 November 2011 / Published online: 6 January 2012

(C) Springer-Verlag 2012

Keywords Cumulative dose - Cumulative exposure

Diabetes and cancer - Insulin glargine

To the Editor: I have with interest read the recent article by Ruiter et al entitled 'Risk of cancer in patients on insulin glargine and other insulin analogues in comparison with those on human insulin: results from a large populationbased follow-up study' [1].

The authors explain in their methods section that they used Cox proportional hazard models with duration of cumulative drug use as a time-varying determinant [1], as described by Stricker and Stijnen [2]. They state that 'In this model, cumulative exposure in participants with cancer at the date of diagnosis is compared with cumulative exposure in all individuals without cancer with the same duration of insulin exposure in days.'

Thus, it seems that the time-varying covariate they use is a variable with time (days on insulin) as units. Yet, they report HRs between insulin glargine (A21Gly,B31Arg, B32Arg human insulin)/other analogues and human insulin. If a covariate in a regression model has a unit, the regression coefficient should be per unit; this is completely unrelated to whether the covariate is time-dependent or not. So it seems

B. Carstensen $(\bowtie)$

Steno Diabetes Center,

Niels Steensens Vej 2,

2829 Gentofte, Denmark

e-mail: bxc@steno.dk that the unit is missing in the reporting. Are the HRs reported actually HRs per year of exposure?

If we make this assumption, then the reported HR for insulin glargine of 0.7 (Table 2) refers to the HR comparing a person on insulin glargine for 1 year with a person on human insulin for 1 year. The comparison after 2 years would then be a HR of $0.7^{2}$, etc. And similarly for the comparison of the other analogues with human insulin.

This is, however, a minor point relative to the hidden assumption of equality of risks at initiation. If the variables really are coded as cumulative (years?) on a particular insulin analogue, and there are no intercepts (indicator of current treatment) in the model, then the authors assume that there is no initial difference between the three groups of patients allocated human insulin, insulin glargine or other analogues. This is tantamount to analysing data as if they came from a randomised trial, and would almost certainly give biased results owing to confounding by indication.

An alternative explanation of the reporting of results may be that the analyses only included the variable 'currently on insulin glargine' (and 'currently on other analogues'), leaving Table 2 correct but the description of the methods incorrect. This would also be in line with the paper by Stricker and Stijnen [2], which only discusses time-dependent variables in the form of indicator functions and not as cumulative exposures at all. In that case the HR would refer to HRs that were constant at any time after initiation, independent of cumulative exposure. An analysis that would partly control 
for confounding by indication but, on the other hand, would not allow for cumulative exposure effects.

It would be most welcome to have the authors' clarification of which model was actually used, that is, whether it is a constant HR of 0.7 or a HR of 0.7 per year of insulin glargine exposure, i.e. a decreasing $\mathrm{HR}$ with increasing duration of use, and whether the patients were assumed to be at the same risk of cancer at the initiation of treatment.

Contribution statement The author was responsible for the conception and design of the manuscript, wrote the manuscript and approved the final version for publication.
Duality of interest B. Carstensen is a stockholder of NovoNordisk and Senior Statistician at Steno Diabetes Center, a research hospital owned by NovoNordisk.

\section{References}

1. Ruiter R, Visser LE, van Herk-Sukel MPP et al (2012) Risk of cancer in patients on insulin glargine and other insulin analogues in comparison with those on human insulin: results from a large population-based follow-up study. Diabetologia. doi:10.1007/ s00125-011-2312-4

2. Stricker BH, Stijnen T (2010) Analysis of individual drug use as a time-varying determinant of exposure in prospective populationbased cohort studies. Eur J Epidemiol 25:245-251 